PAPER

\title{
Predictors and prognosis of refractory status epilepticus treated in a neurological intensive care unit
}

\author{
M Holtkamp, J Othman, K Buchheim, H Meierkord
}

J Neurol Neurosurg Psychiatry 2005;76:534-539. doi: 10.1136/jnnp.2004.041947

See end of article for authors' affiliations

.....................

Correspondence to:

Dr M Holtkamp

Department of Neurology, Charité-

Univeristätsmedizin Berlin,

Schumannstr. 20/21,

10117 Berlin, Germany;

martin.holtkamp@charite.

de

Received 22 March 2004

In revised form

14 July 2004

Accepted 15 July 2004

\begin{abstract}
Objective: To assess risk factors and prognosis in patients with refractory status epilepticus (RSE).
Methods: We retrospectively analysed all episodes of status epilepticus (SE) treated between 1993 and 2002 on the neurological intensive care unit (NICU) of the Charité-Universitätsmedizin Berlin. The predictive and prognostic features of RSE were compared with non-RSE (NRSE). All patients with "de novo" SE were followed up to identify the possible development of post-SE symptomatic epilepsy.

Results: A total of 83 episodes fulfilled our criteria of SE. Of these $43 \%$ were refractory to first line anticonvulsants. The mean age of patients with SE was 53.3 (SD 19) years, with only two patients younger than 18 years. Encephalitis was significantly more often the primary cause in RSE ( $<<0.05)$, whereas low levels of antiepileptic drugs were significantly more often associated with NRSE $(p<0.001)$. Hyponatraemia within the first 24 hours after onset of status activity was significantly more often associated with RSE $(p<0.05)$. In RSE, compared with NRSE, significantly longer duration of seizure activity $(p<0.001)$, more frequent recurrence of epileptic activity within the first 24 hours after the end of seizure activity $(p<0.001)$, longer stay in the NICU and in hospital ( $p<0.001$ and $p<0.01$, respectively), and more frequent development of symptomatic epilepsy $(p<0.05)$ were seen.

Conclusions: SE treated in the NICU is frequently refractory to first line anticonvulsant drugs. Encephalitis is a predictor for RSE, which is associated with markedly poor outcome, in particular, the development of post-SE symptomatic epilepsy. Thus prevention of this most severe form of SE should be the primary target of treatment of SE.
\end{abstract}

$\mathrm{R}$ efractory status epilepticus (RSE) is a condition in search of improved clinical characterisation and more efficient treatment options. In contrast with status epilepticus (SE) in general, only a few studies have been reported on the subgroup of refractory status. There are as yet no reliable incidence figures for RSE. However, estimates have suggested that $30-50 \%$ of all cases of status are refractory to first line anticonvulsants. ${ }^{12}$ Given the high incidence of SE-that is, $10-41 / 100000,{ }^{3-8}$ the extent of the problem of refractory status becomes obvious. Despite its frequency, little is known about the predictive and prognostic features of the critical condition of RSE.

The problem is complicated further by the fact that the definitions too are unclear. While the definition of SE, which has so far been based on the 30 minute period, is currently being redefined, ${ }^{9}{ }^{10}$ there is no generally accepted definition for RSE. Some authors apply a minimum duration of seizure activity, ${ }^{11}{ }^{12}$ while others only refer to the failure of two or three anticonvulsants independent of the time span that has elapsed since onset. ${ }^{13-15}$

Identification of predictors for RSE is crucial for detection of patients at risk early in the course of the disease. Thus, tailoring the treatment escalation strategies for such patients may, on the one hand, prevent adverse effects, and on the other hand, reduce the risk for developing long term RSE including its deleterious consequences. So far, a retrospective cohort study has suggested that the semiology of status may contain predictive information, with non-convulsive SE and focal motor status seen significantly more often in RSE compared with non-RSE (NRSE), ${ }^{1}$ although the primary cause of SE did not have a significant impact on refractoriness.

Several studies have assessed outcome in SE in general. Mortality within 30 days (short term mortality) after SE has been described as between $7 \%$ and 39\%. ${ }^{4716}$ Morbidity including severe focal neurological deficits, cognitive impairment, and development of epilepsy is seen in $3-13 \%$ of cases. ${ }^{8}{ }^{17-21}$ However, systematic prognostic data focusing on RSE are generally lacking. In particular, rates of post-SE symptomatic epilepsy are largely unknown and, so far, have not been analysed in detail.

The aim of the present study was to identify risk factors and outcome in RSE. We compared retrospectively the predictive and prognostic features of episodes of RSE with those of NRSE in patients treated in a neurological intensive care unit (NICU) over a period of 10 years. Furthermore, we analysed and compared the development of post-SE symptomatic epilepsy in the two groups.

\section{PATIENTS AND METHODS}

\section{Definitions and classifications}

- Status epilepticus: Our definition of SE included all semiological forms of clinical and electrophysiological epileptic activity lasting more than five minutes or recurrent epileptic activity over a period of more than five minutes without regain of the pre-existing level of consciousness (the latter part of the definition does not apply to simple partial SE). We thus adopted the time window suggested in the operational definition of Lowenstein et al ${ }^{9}$ and extended this to all forms of SE.

- Refractory status epilepticus: RSE was defined as status that does not respond to initial anticonvulsant treatment with benzodiazepines and phenytoin regardless of the delay since the onset of the seizure. Duration of seizure activity

Abbreviations: AED, antiepileptic drug; CNS, central nervous system; CPSE, complex partial status epilepticus; GCSE, generalised convulsive $\mathrm{SE}$; NICU, neurological intensive care unit; RSE, refractory status epilepticus 
as a major part of the definition does not appear to be very helpful since treatment escalation after failure of first line anticonvulsants in other than generalised convulsive forms of SE is usually not done instantly. ${ }^{22}$ First line anticonvulsant drugs have to be given in the appropriate form and in adequate dosages. An acute anticonvulsant treatment regimen with first line drugs was considered adequate if it included intravenous administration of $10 \mathrm{mg}$ diazepam, $1 \mathrm{mg}$ clonazepam, $6 \mathrm{mg}$ lorazepam, or $5 \mathrm{mg}$ midazolam followed by $750 \mathrm{mg}$ phenytoin or an analogue dosage of fosphenytoin. SE of patients who did not receive phenytoin or fosphenytoin was defined as refractory with continuing epileptic activity after a dosage of benzodiazepines double that described above.

In comatose patients with none or only subtle motor phenomena, SE was defined by the presence of repetitive generalised or focal epileptiform discharges (spikes, sharp waves, and spike waves) whereas periodic lateralised epileptiform discharges alone were not regarded as diagnostic.

- Pre-existing epilepsy: This was defined as two or more unprovoked epileptic seizures that had occurred at least more than four weeks before the onset of SE.

- Post-SE symptomatic epilepsy: In patients with "de novo" SE-that is, without pre-existing epilepsy, the development of symptomatic epilepsy after SE was defined as the occurrence of at least one unprovoked epileptic seizure occurring not earlier than four weeks after termination of SE.

The term "anticonvulsant" is used to describe drugs administered for the treatment of SE and the term "antiepileptic" is used to describe drugs administered in existing epilepsy.

We used the criteria of the International League against Epilepsy (ILAE) for the clinical classification of SE. ${ }^{10}$ In a first step the causes of SE were subsumed into broader categories, along the lines suggested by Hauser et $a^{23}$ and Hesdorffer et $a l^{8}$ with some modifications:

- central nervous system (CNS) disease (acute symptomatic, progressive, and remote)

- substance associated (intoxication, withdrawal, and low levels of antiepileptic drugs (AEDs))

- idiopathic/cryptogenic.

SE was considered to be caused by "acute symptomatic" CNS disease if it occurred within one week after an acute brain insult. "Progressive" CNS disease was defined as the presence of a non-static CNS condition such as tumour, multiple sclerosis, or a neurodegenerative disease. "Remote" CNS disease was defined as presence of a history of CNS insult, and the time between the SE and the neurological insult had to be more than one week. SE was classified as idiopathic/cryptogenic in the absence of acute, progressive, or remote CNS disease as well as absence of any substance association. ${ }^{8}$ If the SE could be assigned aetiologically to more than one of the subgroups described above, it was assigned to the most probable group after considering the overall clinical picture.

Encephalitis was defined as encephalopathy (depressed or altered level of consciousness lasting $\geqslant 24$ hours, lethargy, or change in personality) and one or more than one of the following symptoms: fever, focal neurological findings, cerebrospinal fluid (CSF) pleocytosis, or electroencephalogram (EEG) or neuroimaging findings consistent with encephalitis. ${ }^{24}$ Encephalitis was classified as infectious, non-infectious, or of unknown aetiology. Infectious brain diseases caused by bacteria or parasites were not included in the diagnosis of encephalitis but were itemised separately. In our NICU, all patients with encephalitis are tested for neurotropic viruses including serological and polymerase chain reaction examinations. They are also tested for enteroviruses, arboviruses, Bartonella species, Chlamydia species, and Mycoplasma pneumoniae including serological examination of serum and CSF.

SE was terminated in patients not treated with anaesthetics on the basis of clinical description and in patients treated with anaesthetics with cessation of seizure activity and absence of burst suppression patterns in the EEG.

\section{Patients}

We retrospectively analysed all episodes of SE treated over a period of 10 years (January 1993 to December 2002) in the NICU at the Charité University Hospital (CharitéUniversitätsmedizin Berlin). To ensure identification of all possible episodes we conducted a computer assisted search of patient files using the keywords "SE", "seizure clustering", and "prolonged epileptic seizures". Episodes were included if SE commenced after admission to the NICU, before admission but still continuing after admission to the NICU, and SE terminated near-term before admission to the NICU, if the admission was causally related to the SE. Episodes were excluded if the records of the patient were not retreivable, if impairment of consciousness between two seizures within five minutes was iatrogenic, or if the episode classified as SE was retrospectively a paroxysmal incident of non-epileptic origin-that is, psychogenic non-epileptic seizure, prolonged convulsive syncope, transient ischaemic attack, etc.

By these criteria we identified 140 episodes in 131 patients, suitable for SE. The records of five patients with six episodes were not located in the archives of the hospital. From the remaining cases, 83 episodes in 79 patients fulfilled our definition of SE. The patients with "de novo" SE were followed up by telephone interview with regard to the development of symptomatic epilepsy after SE. Before the telephone interview patients were contacted by mail giving them the choice to refuse the interview. The local ethics committee of the Universitätsklinikum Charité approved the procedure and informed consent was obtained from the patients taking part in the follow up study.

\section{Clinical data}

To analyse the clinical variables we employed a structured data collection grid that was used by two independent reviewers. For each episode of SE, the patient's demographic data (age, sex) and medical history (acute/chronic, neurological/non-neurological) were documented. Data on one-onone aetiology, semiology, and clinical course were evaluated. In addition, paraclinical data from the first 24 hours after the onset of SE including serum sodium and glucose levels, rectal temperature, and CSF variables were analysed. Finally, we analysed outcome measures including duration of SE, short term reoccurrence of epileptic activity within 24 hours after termination of SE, length of stay in hospital and in the NICU, inhospital mortality, and, in patients with "de novo" SE, development of symptomatic epilepsy. As the prognosis of complex partial SE (CPSE) and generalised convulsive SE (GCSE) may differ, subgroup analysis of these two types of SE with regard to outcome measures was performed.

\section{Statistical analysis}

Data were collected with the help of the database program Microsoft Access 2000. Statistical calculations were performed with SPSS 11.0. Frequency distributions of predictive and prognostic features of RSE and NRSE were compared in order to identify characteristics of RSE and were calculated using the $\chi^{2}$ test. The $t$ test was used for analysis of continuous data with normal distribution and the MannWhitney $\mathrm{U}$ test for data with non-normal distribution. Where 
applicable, Pearson's correlation coefficient was calculated. Differences were considered significant at $\mathrm{p}<0.05$.

\section{RESULTS}

\section{Study population}

A total of 83 episodes in 79 patients $(51 / 83$ (61.4\%) female) treated in the NICU fulfilled our diagnostic criteria for SE. The mean age was 53.3 (SD 19) years (range 11-94) with one peak in the fourth decade and another one in the seventh. The vast majority of patients included in this study were adults, with only two patients 11 and 16 years of age. Following our definition, 36 episodes $(43.4 \%)$ complied with refractory SE (RSE). Distribution of age and sex was not significantly different between the two study groups. SE persisted with admission to the NICU in $50.6 \%(42 / 83)$ and was terminated just before admission in $41 \%(34 / 83)$ of cases. In $8.4 \%(7 / 83)$ of cases SE occurred while patients were treated in the NICU for other reasons.

\section{Comorbidity}

The most common pre-existing diseases in the patients were epilepsy $(33 / 83,39.8 \%)$, arterial hypertension $(20 / 83,24.1 \%)$, cardiovascular disease, chronic alcohol abuse, and manifest stroke $(19 / 83,22.9 \%)$. Pre-existing epilepsy was seen significantly more often in patients with NRSE (24/47, 51.1\%) than in patients with RSE (9/36, 25\%; p<0.05). Cardiovascular disease and stroke were seen more often in RSE, and arterial hypertension and chronic alcohol abuse were seen more often in NRSE, but the differences were not significant.

\section{Aetiology}

SE was caused by diseases affecting primarily the CNS in $88.9 \%$ of refractory cases and $51.1 \%$ of non-refractory cases $(\mathrm{p}<0.001)$. This finding can mainly be attributed to acute symptomatic CNS diseases, which were the cause in $50 \%$ of episodes with RSE and $14.9 \%$ of episodes with NRSE $(\mathrm{p}<0.01)$. Progressive and remote CNS diseases causing SE were not significantly different in the two study groups. In contrast, substance associated SE was seen significantly more often in NRSE (36.2\%) compared with RSE ( $11.1 \%$; p < 0.05$)$. Similarly, idiopathic/cryptogenic SE caused NRSE in $14.9 \%$ of cases and RSE in none $(\mathrm{p}<0.05)$ (table 1$)$.

The individual aetiologies are listed in table 2. In 3/10 cases with encephalitis, neurotrope viruses were proved to be the causative agents, and in $7 / 10$ the aetiology remained unknown. Encephalitis was causative significantly more often in RSE (22.2\%) compared with NRSE $(4.3 \%$; p<0.05). In contrast, insufficient levels of AEDs was the main aetiological factor in NRSE $(27.7 \%)$ but not at all in RSE $(p<0.01)$. In the subgroup of patients with pre-existing epilepsy low levels of AEDs were significantly more often causal for NRSE (54.2\%) compared with RSE $(0 \%)$ as well $(\mathrm{p}<0.001)$.

\begin{tabular}{|c|c|c|c|}
\hline Aetiology & $\begin{array}{l}\text { NRSE } \\
(\mathrm{n}=47)\end{array}$ & $\begin{array}{l}\text { RSE } \\
(n=36)\end{array}$ & $p$ value \\
\hline CNS disease & $23(48.9 \%)$ & 32 (88.9\%) & $<0.001$ \\
\hline Acute symptomatic & $7(14.9 \%)$ & $18(50 \%)$ & 0.001 \\
\hline Unprovoked & $16(34 \%)$ & 14 (38.9\%) & 0.65 (NS) \\
\hline Remote & $10(21.3 \%)$ & $8(22.2 \%)$ & 1.0 (NS) \\
\hline Progressive & $6(12.8 \%)$ & $6(16.7 \%)$ & 0.76 (NS) \\
\hline Substance associated & $17(36.2 \%)$ & $4(11.1 \%)$ & 0.011 \\
\hline Idiopathic/cryptogenic & $7(14.9 \%)$ & 0 & 0.017 \\
\hline
\end{tabular}

NRSE, non-refractory status epilepticus; RSE, refractory status epilepticus; CNS, central nervous system; NS, not significant.

\section{Paraclinical factors}

Serum sodium was measured in 73 episodes within the first 24 hours after onset of SE. Hyponatraemia with serum sodium of less than $135 \mathrm{mmol} / \mathrm{l}$ was seen significantly more often in episodes of RSE (10/29; 34.5\%) compared with episodes of NRSE $(6 / 44,13.6 \% ; p<0.05)$. Low serum sodium did not correlate with aetiology or semiology of SE. Data for serum glucose were available for 68 episodes in the first 24 hours of SE. Hyperglycaemia with serum glucose levels of more than $10 \mathrm{mmol} / \mathrm{l}$ was seen in 3/25 episodes of RSE and in 11/43 episodes of NRSE, the difference was not significant. Temperature was documented in 48 episodes, in a quarter of cases patients had fever with rectal temperatures of more than $38.5^{\circ} \mathrm{C}$. Fever was seen significantly more often in RSE (9/24, 37.5\%) compared with NRSE (3/24, 12.5\%; p<0.05). However, fever correlated significantly positively with acute encephalitis $(r=0.66 ; \mathrm{p}<0.05)$ and thus was not an independent factor. CSF was examined in 46 cases $(55.4 \%)$. Pleocytosis was seen slightly more often in RSE $(8 / 22,36.4 \%)$ than in NRSE $(7 / 24,29.2 \%)$, and white cell count in the CSF was higher in NRSE compared with RSE, but differences were not significant (fig 1).

\section{Semiology}

The most common form of SE was complex partial SE (CPSE; $41 / 83,49.4 \%)$, followed by generalised convulsive SE (GCSE) with partial onset $(16 / 83,19.3 \%)$ and GCSE without partial onset $(15 / 83,18.1 \%)$. Simple partial SE was seen in $8.4 \%$ (7/83) of patients. Absence status and myoclonic status were exhibited by two patients each $(2.4 \%)$, the former was seen in NRSE and the latter in RSE. No form of SE was seen significantly more often in either of our study groups. Taking all forms of focal and focal onset SE together $(64 / 83,77.1 \%)$, focal forms amounted to $77.8 \%(28 / 36)$ in RSE and $76.6 \%$ (36/47) in NRSE.

\section{Outcome measures}

The duration of seizures was significantly longer in RSE (median 92 hours) compared with NRSE (median 2.4 hours; $p<0.001)$. In those patients surviving $S E$, recurrence of epileptic activity within 24 hours after termination of SE was seen significantly more often with RSE (15/33, 45.4\%) compared with NRSE (3/46, 6.5\%; p<0.001) (fig 2). The length of stay in the NICU was significantly longer in RSE (median 16.5 days) compared with NRSE (median 2 days; $p<0.001)$. The length of stay in hospital was also significantly longer in RSE (median 30 days) compared with NRSE (median 10.5 days; $\mathrm{p}<0.01$ ). Inhospital mortality was higher in RSE $(6 / 36,16.7 \%)$ compared with NRSE $(4 / 47,8.6 \%)$, but the difference was not significant. Three patients with RSE and one patient with NRSE died in persisting seizures. The other patients died due to medical complications exclusively.

In 50/83 episodes patients had "de novo" SE and thus were eligible for the follow up telephone interview about development of post-SE symptomatic epilepsy. Seven patients were not followed up because either the current address and telephone number were not ascertainable or the patient refused the interview. Another 26 patients had already died. Thus 17 patients were available for the interview. Nine of these patients had developed symptomatic epilepsy after SE. Symptomatic epilepsy occurred significantly more often after RSE $(7 / 8,87.5 \%)$ compared with NRSE $(2 / 9,22.2 \%$; $\mathrm{p}<0.05)$.

Subgroup analysis of outcome measures with regard to CPSE and GCSE showed that the duration of seizures was significantly longer in CPSE (205.2 hours) compared with GCSE (46.8 hours; $\mathrm{p}<0.05$ ) whereas all other measures were not significantly different between the groups. 
Table 2 Individual aetiologies of status epilepticus

\begin{tabular}{|c|c|c|c|}
\hline Aetiology & $\begin{array}{l}\text { NRSE } \\
(n=47)\end{array}$ & $\begin{array}{l}\text { RSE } \\
(n=36)\end{array}$ & $p$ value \\
\hline \multicolumn{4}{|l|}{ Inflammatory CNS diseases } \\
\hline Encephalitis & $2(4.3 \%)$ & $8(22.2 \%)$ & 0.018 \\
\hline Meningitis & $1(2.1 \%)$ & 0 & 1.0 \\
\hline Multiple sclerosis & $1(2.1 \%)$ & $3(8.3 \%)$ & 0.31 \\
\hline Cerebral toxoplasmosis & $1(2.1 \%)$ & 0 & 1.0 \\
\hline \multicolumn{4}{|l|}{ Cerebrovascular CNS diseases } \\
\hline Acute stroke & 0 & $3(8.3 \%)$ & 0.78 \\
\hline Remote stroke & $7(14.9 \%)$ & $4(11.1 \%)$ & 0.75 \\
\hline Intracerebral haemorrhage & $2(4.3 \%)$ & $2(5.5 \%)$ & 1.0 \\
\hline Sinus venous thrombosis & 0 & $2(5.5 \%)$ & 0.19 \\
\hline \multicolumn{4}{|l|}{ Secondary brain damage } \\
\hline Infantile brain damage & $1(2.1 \%)$ & $1(2.8 \%)$ & 1.0 \\
\hline Hypoxic brain damage & 0 & $3(8.3 \%)$ & 0.78 \\
\hline \multicolumn{4}{|l|}{ Trauma } \\
\hline Post-traumatic brain damage & $2(4.3 \%)$ & 0 & 0.5 \\
\hline \multicolumn{4}{|l|}{ Neoplasia } \\
\hline Primary brain tumour & $2(4.3 \%)$ & $1(2.8 \%)$ & 1.0 \\
\hline Cerebral metastasis & $3(6.4 \%)$ & $2(5.5 \%)$ & 1.0 \\
\hline \multicolumn{4}{|l|}{ Cortical developmental malformation } \\
\hline Cortical dysplasia & 0 & $1(2.8 \%)$ & 0.43 \\
\hline \multicolumn{4}{|l|}{ Encephalopathy } \\
\hline Encephalopathy in hyperammonaemia & 0 & $1(2.8 \%)$ & 0.43 \\
\hline Hypertensive encephalopathy & $1(2.1 \%)$ & $1(2.8 \%)$ & 1.0 \\
\hline \multicolumn{4}{|l|}{ Substance associated } \\
\hline Low levels of AEDs & $13(27.7 \%)$ & 0 & $<0.001$ \\
\hline Alcohol-associated & $3(6.4 \%)$ & $3(8.3 \%)$ & 1.0 \\
\hline Drug associated & $1(2.1 \%)$ & $1(2.8 \%)$ & 1.0 \\
\hline Unknown & $7(14.9 \%)$ & $0(0 \%)$ & 0.017 \\
\hline
\end{tabular}

\section{DISCUSSION}

Status epilepticus (SE) represents one of the most frequent emergency situations in neurology associated with significant morbidity and mortality. ${ }^{621}$ If such status is refractory to first line anticonvulsants the condition becomes even more critical and requires management in an intensive care unit.

Unfortunately, the definition of refractoriness in SE as yet has been subject to much controversy and confusion. In some studies, besides failure of anticonvulsants, a minimum time span that has elapsed since seizure onset ${ }^{111}{ }^{12}$ has been used as the cornerstone of the definition. However, in others the requirement has been the failure of a number of anticonvulsants regardless of duration of seizure activity. ${ }^{13-15}$ In the current study, failure of two anticonvulsants-a benzodiazepine and phenytoin/fosphenytoin-in adequate dosages defined RSE; the duration of status activity was not

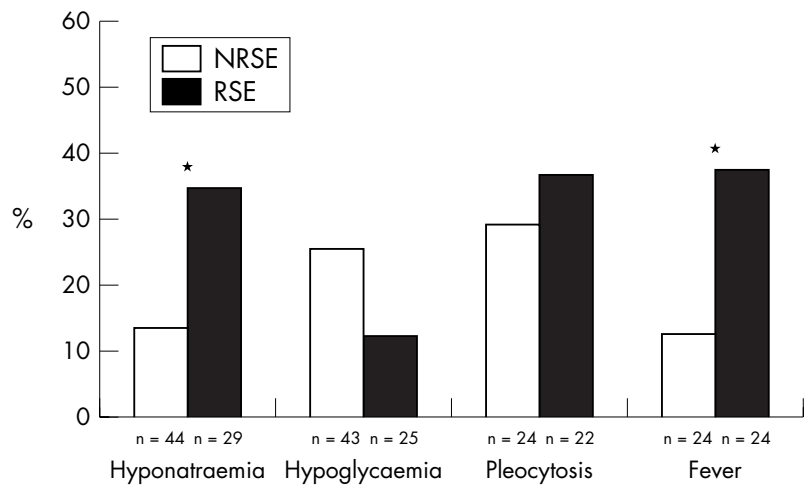

Figure 1 Paraclinical factors measured in the first 24 hours after seizure onset in non-refractory status epilepticus (NRSE) and refractory status epilepticus (RSE). Data are presented as the rate of episodes in each subgroup with the particular feature. Hyponatraemia and fever were seen significantly more often in episodes of RSE. ${ }^{*} p<0.05$. considered. We focused on refractoriness towards anticonvulsants because the time point to escalate anticonvulsant treatment of SE including the administration of anaesthetics is rather variable, with more hesitancy in CPSE compared with GCSE. ${ }^{22}$ Thus a fixed description of status duration as major part of the definition of refractoriness is not convincing, in particular for those cases treated late after the onset of symptoms.

To identify predictive and prognostic characteristics associated with RSE, we analysed data of all patients treated for SE in the NICU at the Charité University Hospital Berlin over a period of 10 years. In addition, patients without preexisting epilepsy were followed up about development of symptomatic epilepsy. Our main findings were: (a) SE was refractory to first line anticonvulsants in $43 \%$ of cases; $(b)$ encephalitis is a major risk factor for RSE; (c) SE caused by

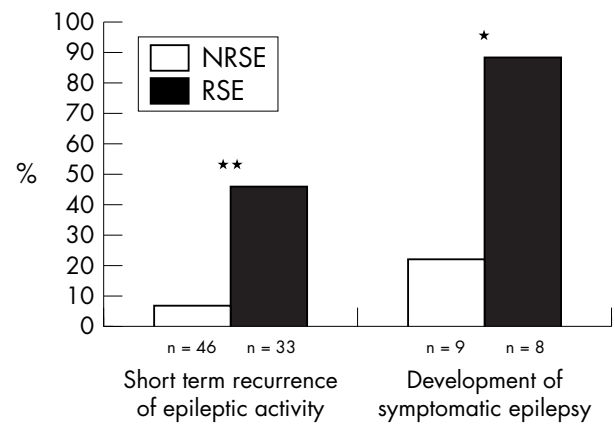

Figure 2 Short term recurrence of epileptic activity in the first 24 hours after seizure termination and development of post status epilepticus symptomatic epilepsy. Data are presented as the rate of episodes in each subgroup (non-refractory status epilepticus (NRSE) and refractory status epilepticus (RSE)) with the particular feature. Short term recurrence of epileptic activity and development of symptomatic epilepsy were seen significantly more offen after RSE. ${ }^{*} \mathrm{p}<0.05 ;{ }^{* *} \mathrm{p}<0.001$. 
insufficient levels of AEDs is usually not refractory; and $(d)$ symptomatic epilepsy develops significantly more often following RSE.

The general demographic features of the population included in this study were similar to a previous retrospective NICU based study on RSE. ${ }^{1}$ As almost all patients were older than 18 years, the clinical features discussed here cannot be considered representative for all age groups. In the present study, the rate of refractory cases was $43 \%$ which is slightly higher than the $31 \%$ reported by Mayer et al. ${ }^{1}$ The differences are most likely due to the definitions used and also perhaps due to differences in the study population. There is a paucity of data on the overall figures of refractory cases and a selection bias might be assumed since patients only from the NICU were considered. Clearly, a number of patients with NRSE will not have entered the NICU. Thus, this series of patients may not be representative of patients with SE in general. It is therefore interesting to note that in a large hospital based trial a very similar rate of $35-44 \%$ of patients did not respond to common initial anticonvulsants. ${ }^{2}$ Thus, the number of refractory cases in the current population does not greatly differ from the hospital based population.

The clinical characteristics of refractory status and risk factors of the condition are poorly understood, therefore current management approaches are still unsatisfactory. ${ }^{22}$ The current study identified acute symptomatic CNS diseases and particularly encephalitis as etiological predictors for RSE. Encephalitis seems to be notably epileptogenic and has been described in previous retrospective case series to cause RSE requiring long term treatment with anticonvulsant anaesthetics, sometimes up to several weeks. ${ }^{12} 2526$ Pathogenetically, the multifocal distribution of the cortical epileptogenic lesions as caused by encephalitis ${ }^{27}$ is likely to represent an important factor predisposing to refractoriness in SE. Furthermore, some causative agents such as herpes virus, predominantly affect the temporal lobe, ${ }^{27}$ a structure well known for low seizure threshold. ${ }^{28}{ }^{29}$ The current data suggest an aggressive therapeutic approach with rapid escalation of treatment in SE associated with encephalitis. This may prevent functional and structural neurological deficits resulting from continuing seizure activity itself ${ }^{30}$ which add to the deficits emerging in the course of encephalitis.

In contrast, insufficient levels of AEDs in patients with preexisting epilepsy were not seen at all in the RSE group, but this was the most common cause of NRSE. It has been shown previously that patients with SE caused by insufficient levels of AEDs have better prognosis with lower mortality. ${ }^{31}$ Our current findings are important as various authors have shown that low levels of AEDs is one of the most common aetiologies in SE in general. ${ }^{8} 203132$ With respect to therapeutic management in clinical practice, treatment escalation in SE caused by insufficient levels of AEDs should be used reluctantly.

Clinical and experimental data have shown the proconvulsive properties of hyponatraemia. ${ }^{33-35}$ In the current study, hyponatraemia measured in the first 24 hours after onset of SE was significantly associated with refractoriness. These patients had not been exposed to carbamazepine, oxcarbazepine, or other drugs possibly causing low serum sodium levels significantly more often compared with patients with NRSE. Furthermore, hyponatraemia did not correlate with the primary cause of SE. As SE is not known to cause low serum sodium levels, the current results may support previously reported proconvulsive properties of hyponatraemia maintaining SE. Although the causal relationship between hyponatraemia and RSE is as yet unclear, the current findings suggest balancing hyponatraemia in all patients with SE with due consideration of the risk of severe neurological deficits when reloading serum sodium too rapidly.

The overall clinical outcome is poor in RSE. Similar findings have been described before in the general condition of SE of long duration. ${ }^{19}$ However, the specific clinical consequences of RSE episodes compared with NRSE, as shown in the current study, reveal a significant association with poor outcome and post-SE symptomatic epilepsy.

Symptomatic epilepsy frequently follows SE in patients who have previously not had epileptic seizures. ${ }^{18}$ The development of epilepsy in such circumstances is usually associated with the cerebral damage that has taken place. However, in most cases it is difficult to determine if such damage is related to the underlying pathology, to the status as such, or to a combination of both. ${ }^{36-38}$ Experimental animal data indicate that symptomatic epilepsy in the wake of status is not necessarily associated with major structural lesions but may also result from plastic changes of the brain that may not be detected with current imaging modalities. ${ }^{39}$

To our knowledge, the effects of NRSE and RSE on the development of symptomatic epilepsy have not yet been analysed. The current data clearly show that refractory status is significantly more often followed by symptomatic epilepsy. This finding was independent of the type of SE. The current data suggest at least a prominent contribution of SE itself to the process of epileptogenesis. The influence of continuing epileptic activity on development of symptomatic epilepsy is also supported by the finding that the 10 year cumulative incidence of later unprovoked seizures is 13\% after acute symptomatic seizures and $42 \%$ after acute symptomatic SE. ${ }^{8}$ However, it has to be considered that the neuronal lesions caused by severe acute symptomatic CNS diseases rather result in RSE and in more frequent unprovoked seizures.

More than half of the patients in the current study who had been followed up for possible development of symptomatic epilepsy died. Most of these patients died in other hospitals and the causes of death remain obscure. Follow up studies looking specifically into mortality and causes of death following RSE will be of great interest.

In summary, RSE is a frequent condition and patients with encephalitis as primary cause of SE are at special risk of developing RSE. Our data suggest treatment escalation early in the course of SE in these patients. Hyponatraemia early in the course of SE may facilitate the development of refractoriness, thus cautious balancing of serum sodium levels may be recommended. Prevention of the refractory type of SE is vital as this condition is associated with markedly poor prognosis including frequent short term recurrences of seizure activity, increased length of stay in the ICU and in hospital, and frequent development of post-SE symptomatic epilepsy.

\section{Authors' affiliations \\ M Holtkamp, J Othman, K Buchheim, H Meierkord, Department of Neurology, Charité-Universitätsmedizin Berlin, Berlin, Germany \\ Competing interests: none declared}

\section{REFERENCES}

1 Mayer SA, Claassen J, Lokin J, et al. Refractory status epilepticus: frequency, risk factors, and impact on outcome. Arch Neurol 2002;59:205-10.

2 Treiman DM, Meyers PD, Walton NY, et al. A comparison of four treatments for generalized convulsive status epilepticus. Veterans Affairs Status Epilepticus Cooperative Study Group. N Engl J Med 1998;339:792-8.

3 Shorvon S. Tonic clonic status epilepticus. J Neurol Neurosurg Psychiatry 1993;56:125-34

4 Knake S, Rosenow F, Vescovi M, et al. Incidence of status epilepticus in adults in Germany: a prospective, population-based study. Epilepsia 2001:42:714-18.

5 DeLorenzo RJ, Hauser WA, Towne AR, et al. A prospective, population-based epidemiologic study of status epilepticus in Richmond, Virginia. Neurology 1996;46:1029-35. 
6 Vignatelli L, Tonon C, D'Alessandro R. Incidence and short-term prognosis of status epilepticus in adults in Bologna, Italy. Epilepsia 2003;44:964-8

7 Coeytaux A, Jallon P, Galobardes B, et al. Incidence of status epilepticus in French-speaking Switzerland: (EPISTAR). Neurology 2000;55:693-7.

8 Hesdorffer DC, Logroscino G, Cascino G, et al. Incidence of status epilepticus in Rochester, Minnesota, 1965-1984. Neurology 1998;50:735-41.

9 Lowenstein DH, Bleck T, Macdonald RL. It's time to revise the definition of status epilepticus. Epilepsia 1999;40:120-2.

10 Commission on Classification and Terminology of the International League against Epilepsy. Proposal for revised clinical and electroencephalographic classification of epileptic seizures. From the Commission on Classification and Terminology of the International League Against Epilepsy. Epilepsia 1981;22:489-501.

11 Stecker MM, Kramer TH, Raps EC, et al. Treatment of refractory status epilepticus with propofol: clinical and pharmacokinetic findings. Epilepsia 1998;39:18-26.

12 Prasad A, Worrall BB, Bertram EH, et al. Propofol and midazolam in the treatment of refractory status epilepticus. Epilepsia $2001 ; 42: 380-6$.

13 America's Working Group on Status Epilepticus. Treatment of convulsive status epilepticus. Recommendations of the Epilepsy Foundation of America's Working Group on Status Epilepticus. JAMA 1993;270:854-9.

14 Cascino GD. Generalized convulsive status epilepticus. Mayo Clin Proc 1996;71:787-92.

15 Lowenstein DH, Alldredge BK. Status epilepticus. N Engl J Med 1998;338:970-6

16 Logroscino G, Hesdorffer DC, Cascino G, et al. Short-term mortality after a first episode of status epilepticus. Epilepsia 1997;38:1344-9.

17 Shneker BF, Fountain NB. Assessment of acute morbidity and mortality in nonconvulsive status epilepticus. Neurology 2003;61:1066-73.

18 Aicardi J, Chevrie JJ. Convulsive status epilepticus in infants and children. A study of 239 cases. Epilepsia 1970;11:187-97.

19 Aminoff MJ, Simon RP. Status epilepticus. Causes, clinical features and consequences in 98 patients. Am J Med 1980;69:657-66.

20 Lowenstein DH, Alldredge BK. Status epilepticus at an urban public hospital in the 1980s. Neurology 1993;43:483-8.

21 Cascino GD, Hesdorffer D, Logroscino G, et al. Morbidity of nonfebrile status epilepticus in Rochester, Minnesota, 1965-1984. Epilepsia, 1998;39:829-32.

22 Holtkamp M, Masuhr F, Harms L, et al. The management of refractory generalised convulsive and complex partial status epilepticus in three European countries: a survey among epileptologists and critical care neurologists. J Neurol Neurosurg Psychiatry 2003;74:1095-9.
23 Hauser WA Anderson VE, Loewenson RB, et al. Seizure recurrence after a first unprovoked seizure. N Engl J Med 1982;307:522-8

24 Glaser CA, Gilliam S, Schnurr D, et al. In search of encephalitis etiologies: diagnostic challenges in the California Encephalitis Project, 1998-2000. Clin Infect Dis 2003:36:731-42.

25 Yaffe K, Lowenstein DH. Prognostic factors of pentobarbital therapy for refractory generalized status epilepticus. Neurology 1993;43: 895-900.

26 Parent JM, Lowenstein DH. Treatment of refractory generalized status epilepticus with continuous infusion of midazolam. Neurology 1994;44: 1837-40.

27 Kennedy PG, Chaudhuri A. Herpes simplex encephalitis. J Neurol Neurosurg Psychiatry 2002;73:237-8.

28 Falconer MA, Serafetinides EA, Corsellis JA. Etiology and pathogenesis of temporal lobe epilepsy. Arch Neurol 1964;10:233-48.

29 Vadlamudi L, Scheffer IE, Berkovic SF. Genetics of temporal lobe epilepsy. J Neurol Neurosurg Psychiatry 2003;74:1359-61.

30 Meldrum BS, Vigouroux RA, Brierley JB. Systemic factors and epileptic brain damage. Prolonged seizures in paralyzed, artificially ventilated baboons. Arch Neurol 1973;29:82-7.

31 Towne AR, Pellock JM, Ko D, et al. Determinants of mortality in status epilepticus. Epilepsia 1994;35:27-34.

32 Sagduyu A, Tarlaci S, Sirin H. Generalized tonic-clonic status epilepticus: causes, treatment, complications and predictors of case fatality. J Neurol 1998; $245: 640-6$

33 Farrar HC, Chande VT, Fitzpatrick DF, et al. Hyponatremia as the cause of seizures in infants: a retrospective analysis of incidence, severity, and clinical predictors. Ann Emerg Med 1995;26:42-8.

34 Sterns RH, Thomas DJ, Herndon RM. Brain dehydration and neurologic deterioration after rapid correction of hyponatremia. Kidney Int 1989;35:69-75.

35 Barolomei F, Gastaut JL. Complex partial status epilepticus provoked by hyponatremia. Eur Neurol 1998;40:53-4.

36 Herman ST. Epilepsy after brain insult: targeting epileptogenesis. Neurology 2002;59:S21-S26.

37 Shorvon S. Does convulsive status epilepticus (SE) result in cerebral damage or affect the course of epilepsy-the epidemiological and clinical evidence? Prog Brain Res 2002;135:85-93.

38 Lowenstein DH. Status epilepticus: an overview of the clinical problem. Epilepsia 1999;40(suppl 1):S3-S8.

39 Loscher W. Animal models of epilepsy for the development of antiepileptogenic and disease-modifying drugs. A comparison of the pharmacology of kindling and post-status epilepticus models of temporal lobe epilepsy. Epilepsy Res 2002;50:105-23. 